\title{
Biomarkers in Colorectal Cancer Screening
}

\author{
Minhhuyen T. Nguyen, MD, and David S. Weinberg, MD, MSc
}

\begin{abstract}
Colorectal cancer (CRC) is the third most common cause of cancer death in men and women in the United States. The main goals of screening are to prevent carcinogenesis (via adenoma detection and removal) and detect cancer at an early, curable stage. CRC mortality is steadily dropping in the United States, partly because of greater screening utilization. However, nearly 1 in 3 average-risk people are not up to date with standard CRC screening recommendations. This review surveys a wide range of CRC biomarkers in various stages of development, which may offer attractive risk stratification tools; a few have reached the commercial stage. If widely accepted, these tools may contribute to shift CRC screening practices away from 1-step colonoscopy to a 2-step risk stratification process of predictive biomarker measurements followed by colonoscopy for lower-risk patients with a positive result. Such strategies could potentially increase the rate of CRC screening.

J Natl Compr Canc Netw 2016;14(8):1033-1040
\end{abstract}

\section{NCCN: Continuing Education}

\section{Accreditation Statement}

This activity has been designed to meet the educational needs of physicians and nurses involved in the management of patients with cancer. There is no fee for this article. No commercial support was received for this article. The National Comprehensive Cancer Network (NCCN) is accredited by the ACCME to provide continuing medical education for physicians.

NCCN designates this journal-based CME activity for a maximum of 1.0 AMA PRA Category 1 Credit $^{\mathrm{TM}}$. Physicians should claim only the credit commensurate with the extent of their participation in the activity.

NCCN is accredited as a provider of continuing nursing education by the American Nurses Credentialing Center's Commission on Accreditation.

NCCN designates the education activity for a maximum of 1.0 contact hour. Accreditation as a provider refers to recognition of educational activities only; accredited status does not imply endorse- ment by NCCN or ANCC of any commercial products discussed/ displayed in conjunction with the educational activity. Kristina M. Gregory, RN, MSN, OCN, is our nurse planner for this educational activity.

All clinicians completing this activity will be issued a certificate of participation. To participate in this journal CE activity: 1) review the learning objectives and author disclosures; 2) study the education content; 3) take the posttest with a $66 \%$ minimum passing score and complete the evaluation at http://education.nccn.org/ node/79280; and 4) view/print certificate.

Release date: August 10, 2016; Expiration date: August 10, 2017

\section{Learning Objectives}

Upon completion of this activity, participants will be able to:

- Describe advances in biomarker technology as risk stratification tools for CRC screening

- Evaluate the efficacy, safety, convenience, and cost of stool, imaging, and endoscopy-based CRC screening methods
From Fox Chase Cancer Center-Temple Health, Philadelphia, Pennsylvania. Submitted March 7, 2016; accepted for publication June 8, 2016.

The authors have disclosed that they have no financial interests, arrangements, affiliations, or commercial interests with the manufacturers of any products discussed in this article or their competitors.

Correspondence: Minhhuyen T. Nguyen, MD, Fox Chase Cancer Center, 333 Cottman Avenue, Philadelphia, PA 19111.

E-mail: Minhhuyen.Nguyen@fccc.edu
EDITOR

Kerrin M. Green, MA, Assistant Managing Editor, JNCCN-Journal of the National Comprehensive Cancer Network

Ms. Green has disclosed that she has no relevant financial relationships.

\section{CE AUTHORS}

Deborah J. Moonan, RN, BSN, Director, Continuing Education, has disclosed that she has no relevant financial relationships.

Kristina M. Gregory, RN, MSN, OCN, Vice President, Clinical Information Operations, has disclosed that she has no relevant financial relationships. Rashmi Kumar, PhD, Senior Manager, Clinical Content, has disclosed that she has no relevant financial relationships.

Susan D. Darlow, PhD, Oncology Scientist/Medical Writer, has disclosed that she has no relevant financial relationships. 
Colorectal cancer (CRC) is the third most common cause of cancer death in men and women in the United States. ${ }^{1}$ The lengthy process of adenoma-tocarcinoma progression facilitates the positive impact of CRC screening through 2 mechanisms: (1) prevention of cancer through adenoma detection and subsequent removal, and (2) detection of CRCs that are typically at an earlier stage than those detected mainly by symptoms. Various screening strategies, including fecal occult blood testing (FOBT), sigmoidoscopy, or colonoscopy, reduce CRC incidence by $52 \%$ to $81 \%$ and mortality by $65 \%$ to $84 \%$. $^{2}$ Screen-detected CRC has an approximately $90 \%$ 5-year survival rate. ${ }^{3}$

Despite improving over time, only $65 \%$ of the average-risk US population is regularly in compliance with screening recommendations. ${ }^{4}$ The ideal cancer screening test would be effective, safe, convenient and inexpensive. No currently available stool, imaging, or endoscopy-based method of CRC screening fits all of these criteria. Because colonoscopy is relatively expensive and carries more risks than other screening tests, models have been developed to stratify CRC risk in asymptomatic individuals. ${ }^{5}$ These scores identify lower-risk patients for 2-step screening strategies (positive biomarkers then colonoscopy) and higher-risk patients for direct 1-step colonoscopy. Advances in biotechnology offer an array of methods to detect minute amounts of potentially relevant DNA, RNA, or protein disease markers in various bodily fluids in asymptomatic patients. This review explores how some of these new methods might serve as risk stratification tools relevant to CRC screening.

\section{Stool-Based Biomarkers Hemoglobin Detection}

Despite their low sensitivity $(30 \%-50 \%)$ and relatively low specificity (80\%-90\%) for advanced polyps and cancers, stool guaiac-based tests (gFOBT) are still widely used. ${ }^{6}$ Their simplicity, low cost, and demonstrated benefit when used programmatically make gFOBT an alternative included in all national and international CRC screening guidelines. Fecal immunochemical testing (FIT), which uses a different reaction to specifically identify human hemoglobin from the lower gastrointestinal tract ${ }^{7}$ in stool, has largely supplanted gFOBT because of increased sensitivity without loss of specificity. ${ }^{6}$ For CRC, the overall sensitivity was $89 \%$ with a specificity of $91 \%$ based on a FIT cutoff value of $20 \mathrm{mcg} / \mathrm{g} .{ }^{8}$ Generally, patients prefer FIT to gFOBT because only one stool sample is required and no diet or medication alterations are needed. ${ }^{7}$ FIT results can be quantified, which is useful when colonoscopy follow-up capability is limited.

The sensitivity of FIT is higher for distal cancers than proximal ones and is lower than that of colonoscopy. ${ }^{9,10}$ Although FIT performed less well than colonoscopy in the detection of advanced adenomas, its greater acceptance rate by patients may offset this difference. ${ }^{11,12}$ Patient choice is an important contributor to screening compliance. Overall, screening rates are highest when patients self-select a screening method (FOBT vs colonoscopy) versus when they are prescribed only one option. ${ }^{13}$

\section{Genetics of CRC}

Other types of fecal biomarkers-DNAs, RNAs, and nonheme proteins - have been evaluated as test components to identify colonic neoplasia. So far, only DNA biomarkers have been extensively tested and approved for clinical use.

To interpret the results of fecal DNA tests requires an understanding of the genetic and epigenetic changes that drive colorectal carcinogenesis. Stool DNA testing is based on the detection of genetic and epigenetic changes in tumor cells that exfoliate into the mucocellular layer in the colonic lumen. ${ }^{14,15}$ There are at least 3 distinct pathways to colon cancer, each with genetic and epigenetic components: the chromosomal instability pathway (CIN) and the microsatellite instability (MSI) pathway, both of which demonstrate the standard adenomacarcinoma transformation; and the serrated polyp or $\mathrm{CpG}$ island methylator phenotype (CIMP) pathway. This third pathway explains why some polyps, typically in the right colon, progress from sessile serrated adenoma (SSA) to carcinoma.

Most CRC (65\%-70\%) arises via the CIN pathway, with adenomas as precursor lesions. The pathway is characterized by defects in chromosomal segregation, telomere stability, and the DNA damage response, leading to chromosomal imbalance (aneuploidy) and a high frequency of loss of heterozygosity (LOH). In 1990, Vogelstein et $\mathrm{a}^{16}$ first proposed a specific sequence of mutations in the APC, Kras, and p53 genes in colon carcinogenesis. However, mul- 
Biomarkers in Colorectal Cancer Screening

tiple alternative CIN pathways exist involving even more complex and heterogeneous patterns of gene mutations. ${ }^{17}$

MSI reflects the loss of DNA mismatch repair capacity and is found in $10 \%$ to $15 \%$ of all CRCs. In Lynch syndrome (hereditary nonpolyposis colorectal cancer), mutations in crucial DNA repair enzyme genes, such as MLH1, MSH2, MSH6, PMS2, or EPCAM, can allow DNA copy errors to go uncorrected. These errors will sometimes affect growth-regulating genes, leading to cancer development.

SSAs are the precursor lesions of the serrated neoplasia pathway. This pathway develops through the accumulation of insertion or deletion mutations throughout the genome, leading to genomic instability, which in most cases leads to MSI-high (MSI-H) adenocarcinomas. ${ }^{18}$ The BRAF mutation and hypermethylated SLC5A8 play crucial roles. Progressive loss of cell cycle control and decreased and altered cytoplasmic differentiation produce the characteristic morphologic changes of SSAs. ${ }^{19}$

Despite attempts to delineate separate pathways for CRC, overlapping features of multiple pathways are often found in one tumor. ${ }^{20,21}$ Mutant genes such as APC and TGFBR2/SMAD4 are common in most CRCs, suggesting a central role in colon carcinogenesis. ${ }^{15}$

\section{Epigenetics of CRC}

Epigenetics broadly refers to heritable alterations in gene expression not due to DNA mutations. Together with gene mutations, epigenetic mechanisms are common contributors to the formation of colon polyps and cancer. Epigenetic mechanisms currently believed to be important in CRC are (1) DNA methylation of cytosine bases in $\mathrm{CpG}$ Islands; (2) posttranslational modifications of histones, which are proteins tightly interwoven with DNA to form chromatin; (3) microRNAs (miRNAs) and other noncoding RNAs; and (4) nucleosome positioning. ${ }^{22}$

Aberrant DNA methylation, the most extensively studied form of epigenetic deregulation, is found in virtually all CRCs. The average CRC methylome has thousands of abnormally methylated genes. Common epigenetic alterations in APC, CTNNB1, KRAS, BRAF, SMAD4, TGFBR2, TP53, PIK3CA, ARID1A, SOX9, FAM123B, and ERBB2 genes disturb key signaling pathways or affect genes that regulate DNA repair and cellular proliferation. ${ }^{23,24}$ Epigenetic changes appear to occur very early in the polyp-to-cancer sequence, making them ideal screening biomarkers. In addition, these epigenetic alterations can occur in histologically normal-appearing mucosa, offering the potential for early identification of higher-risk individuals..$^{25}$

\section{Stool DNA Methylation Markers Alone}

Stool DNA methylation biomarkers have multiple advantages over similar blood biomarkers. Aberrant DNA methylation in the blood may signal the presence of different cancers at the same time. ${ }^{26}$ In addition, early CRC or precursor lesions may not yet release markers into the bloodstream. Thus, fecal DNA markers may be more useful in CRC screening. Methylated fibrillin-1 (mFBN1) ${ }^{26}$ and vimentin $(\text { VIM })^{27}$ in feces can be identified in early-stage CRC. In addition, numerous hypermethylated genes have been detected in human stool more readily than from blood samples. Methylated progesterone receptor ( $m P G R)$ and $\mathrm{O}^{6}$-methylguanine-DNA methyltransferase (mMGMT) are 2 examples of exclusive fecal biomarkers. ${ }^{26}$

\section{Stool DNA Mutation Markers Alone}

The initial proof-of-concept study looking for abnormalities in exfoliated cells collected from stool samples used a DNA panel with 21 mutations ( 3 in Kras, 10 in APC, and 8 in p53) along with the MSI marker BAT-26 and a marker (long DNA) thought to reflect disordered neoplastic apoptosis. ${ }^{28}$ When compared with gFOBT, the fecal DNA panel outperformed by detecting advanced colonic lesions, including cancer in $40.8 \%$ versus $14.1 \%(P<.001)$ of cases without compromising specificity (95\%). Despite this difference, most neoplastic lesions identified by colonoscopy were not detected by either noninvasive test.

\section{Stool Multitarget Marker Panel}

Two large multicenter case-control studies evaluated multitarget stool DNA (MT-sDNA) performance. The first-generation prototype test detected mutant KRAS; aberrantly methylated VIM, NDRG4, BMP3, and TFPI2; $\beta$-actin (reference gene for human DNA quantity); and hemoglobin. Its sensitivity for earlystage CRC was $87 \%$, and for adenomas it was $54 \%$ to $92 \%$, depending on size. ${ }^{29}$ A later, more-refined automated MT-sDNA, which detected mutant KRAS, aberrantly methylated BMP3 and NDRG4, $\beta$-actin, 
and hemoglobin, has a sensitivity of $95 \%$ to $97 \%$ for CRCs and $57 \%$ to $83 \%$ for adenomas, depending on size. $^{30}$

These favorable results eventually led to FDA approval for the refined MT-sDNA test in 2014, with a 3-year testing interval based on a large crosssectional study in 90 centers in North America. ${ }^{31}$ The overall sensitivity was $93 \%$ for curable CRC, and $42 \%$ to $66 \%$ for adenomas and SSAs, depending on polyp size and grade, with a specificity of $87 \%$ to $90 \%$. Clinically, MT-sDNA outperforms gFOBT/ FIT but is considerably more costly. Currently, there are no firm recommendations about the evaluation of patients with positive results but a negative colonoscopy, or compelling data suggesting that this test is cost-effective compared with alternative screening strategies.

\section{Stool Protein and RNA Tests}

The use of fecal nonheme proteins and RNAs as markers for early detection and prediction of CRC outcome has gained increasing attention. Fecal proteins such as calprotectin $^{32}$ and tumor M2 pyruvate kinase (M2$\mathrm{PK})^{33,34}$ have been studied as CRC markers, but neither has been found to be superior to FIT. These studies have thus far remained at the proof-of-principle level. ${ }^{35}$

\section{Gut Microbiome}

Microbial dysbiosis, a pathologic imbalance in the gut flora, has been implicated in colonic tumorigenesis. Gut bacterial composition in patients with CRC differed significantly from that in healthy patients. ${ }^{36,37}$ Certain types of bacterial species, such as Fusobacterium, Bacteroides, Parvimonas, and Solobacterium, appeared to have a strong co-occurrence network in patients with CRC. ${ }^{38}$ These microbial networks can be detected across ethnic and international boundaries. Some of their genes were enriched in early-stage CRC, suggesting a potential role as CRC biomarkers. ${ }^{38,39}$ A causal relationship between continual alterations in the gut microbiome and CRC development was demonstrated in a colitis-driven colon cancer mouse model. ${ }^{40}$ Despite these promising findings, host-bacterial interactions remain ill-defined. Mucosa-associated microbiome and fecal microbiome differ, and currently there is no consensus regarding which one to use in CRC screening.

\section{Blood-Based CRC Biomarkers}

A clinically effective blood test for CRC screening would represent a substantial advance. It could be performed with minimal risk and potentially greater patient acceptance. Like any CRC risk stratification tool, a blood test would reduce over-reliance on colonoscopy, an expensive and riskier procedure. The main problem is to identify molecules that are sensitive and specific enough for CRC detection. Unlike stool-based tests, in which marker detection uses exfoliated cells in feces, detection of abnormal cells and associated markers in the systematic blood supply is more complicated. Blood-based tests require either blood vessel invasion (usually a characteristic of later stage cancers) or phagocytosis by inflammatory cells of neoplasia-derived markers.

\section{DNA Markers}

Aberrant DNA Methylation Markers: Methylated SEPT9 is a frequently studied marker in CRC screening. The gene is a member of the septin family involved in cytokinesis and cell cycle control. Hypermethylation of SEPT9 in adenoma and CRC specimens is confined to one of several $\mathrm{CpG}$ islands of this gene. For CRC detection, its sensitivity was $77 \%$ and specificity was $80 \%,{ }^{41}$ which was not better than FIT. ${ }^{42}$ In 2016, this test was narrowly FDA-approved.

In another example, Pedersen et $\mathrm{a}^{43}$ showed that a combination of $\mathrm{mBCAT} 1$ and $\mathrm{mIKZF} 1$ detected CRC with a sensitivity $77 \%$ (higher than either alone) and a combined specificity of $92.4 \%$. Because BCAT1 and IKZF1 genes are both involved in tumor growth and invasiveness, the degree of their hypermethylation and the frequency of their combined detection closely corresponded to CRC stages I-IV, at 50\%, 68\%, 87\%, and $100 \%$, respectively. ${ }^{43}$

Other DNA Markers: Other circulating tumorrelated molecules, such as aberrant DNA markers, microsatellite alterations markers, and mitochondrial DNA, are currently very difficult to detect against the background of all other circulating DNA,44 especially in early-stage CRC, making them unsuitable as screening biomarkers.

\section{RNA Markers}

Various forms of RNA, such as messenger RNA (mRNA), noncoding miRNA, and long noncoding RNA (lncRNA), are very stable outside of cells and 
Biomarkers in Colorectal Cancer Screening

\begin{tabular}{|c|c|c|c|c|c|}
\hline Test & Type & Preparation & Sensitivity & Specificity & References \\
\hline gFOBT & Stool & No & $30 \%-50 \%$ & $80 \%$ & $6,10,62,63$ \\
\hline FIT & Stool & No & $65 \%-70 \%$ & $85 \%$ & $7-10,12$ \\
\hline Colonoscopy & Procedure & Yes & $95 \%-98 \%$ & $90 \%$ & $44,64,65$ \\
\hline CT colonography & Radiology & Yes & $\begin{array}{l}84 \% \\
(A A \geq 6 \mathrm{~mm})\end{array}$ & $90 \%$ & 66,67 \\
\hline $\begin{array}{l}\text { Low-coherence enhanced } \\
\text { backscattering spectroscopy }\end{array}$ & Procedure & Yes & $\begin{array}{l}88 \% \text { AA } \\
71 \% \text { nAA }\end{array}$ & $72 \%$ & 61 \\
\hline $\begin{array}{l}\text { MT-sDNA (heme, DNA } \\
\text { methylation, and mutation } \\
\text { panel) }\end{array}$ & Stool & No & $\begin{array}{l}85 \% \text { CRC } \\
55 \% \text { AA }\end{array}$ & $\begin{array}{l}90 \% \text { CRC } \\
89 \% \text { AA }\end{array}$ & 31 \\
\hline mRNA 7-gene panel & Blood & No & $78 \%$ & $66 \%$ & 46 \\
\hline miRNA 5-gene panel & Blood & No & $\approx 83 \%-91 \%$ & $\approx 70 \%-95 \%$ & 44 \\
\hline IncRNA 1-gene & Blood & No & N/A & N/A & 44 \\
\hline \multicolumn{6}{|l|}{ DNA methylation } \\
\hline SEPT9 & Blood & No & $77 \%$ & $80 \%$ & 41,42 \\
\hline$B C A T 1 / I K Z F 1$ & Blood & No & $77 \%$ & $92.4 \%$ & 43 \\
\hline VIM & Stool & No & $72 \%-77 \%$ & $83 \%-94 \%$ & 27 \\
\hline
\end{tabular}

Abbreviations: AA, advanced adenoma; CRC, colorectal cancer; FIT, fecal immunochemical testing; gFOBT, guaiac fecal occult blood test; IncRNA, long noncoding RNA; MT-sDNA, multitarget stool DNA; N/A, not applicable; nAA, nonadvanced adenoma; VIM, vimentin.

can be extracted, safely stored, and studied in various bodily fluids. In cancer, RNA expression becomes highly dysregulated, and therefore RNA is more conducive to profiling studies.

The general experimental strategy initially consisted of mRNA expression microarray profiles, followed by validation using real-time quantitative reverse transcription polymerase chain reaction (RTqPCR). This technology is being gradually replaced by next-generation sequencing (NGS). NGS is not only fast and cheaper but it also enables researchers to perform direct RNA sequencing (RNA-seq) to sequence the whole transcriptome (ie, the entire set of coding and noncoding RNAs). ${ }^{44}$

mRNA Markers: In a proof-of-concept study, Tsouma et $\mathrm{al}^{45}$ demonstrated that extracted carcinoembryonic antigen (CEA), cytokeratin 20, and epidermal growth factor receptor (EGFR) transcripts from patients with CRC can indicate disease stage and prognosis.

In 2010, Marshall et $\mathrm{al}^{46}$ introduced a bloodbased 7-gene mRNA biomarker panel as a CRC risk assessment tool. These 7 genes (ANXA3, CLEC4D, LMNB1, PRRG4, TNFAIP6, VNN1, and IL2RB) are derived from a 196-gene expression profile using both patients with CRC at various disease stages and healthy controls, and was later validated in a larger population. The test panel has a sensitivity of $78 \%$ and a specificity of $66 \%$ (Table 1 ), and provides a scale assessing a person's current relative risk of having CRC based on gene signature.

miRNA Markers: The roles of miRNAs in regulating posttranslational gene expression in cancer development, growth, and metastasis have been well-established in a large body of research publications. ${ }^{47} \mathrm{~A}$ single miRNA, a short 18- to 22-nucleotide-long noncoding molecule, can normally target many mRNAs. miRNAs lack complex transcriptional and translational modifications compared with mRNAs and proteins. Almost all cancer types present a unique profile of upregulated and downregulated miRNAs. Thus, these characteristics can potentially make miRNAs very useful cancer biomarkers. ${ }^{47}$ miRNAs can behave as tumor suppressors, oncogenes, or both. ${ }^{48}$

miR-17-3p, miR-92, miR-92a, miR-29a, miR141, and miR-21 have been associated with advanced CRC and poor prognosis. ${ }^{44,49} \mathrm{miR}-21 \mathrm{ex}$ pression levels may be a special pan-cancer marker, because it has been shown to be upregulated in other cancers. ${ }^{50}$ miR-15b, miR-17-3p, miR-18a, miR-20a, miR-21, miR-29a, and miR-92a have been studied by multiple investigators for CRC detection. ${ }^{44}$ 
Nguyen and Weinberg

However, conflicting data limit their usefulness. Methods for specimen collection, processing, purification, and analysis of RNA must be standardized before miRNA tests can serve as reliable cancer biomarkers.

lncRNA Markers: lncRNAs, molecules 200 nucleotides or longer, regulate gene expression and cellular functions. ${ }^{51}$ They appear to play a role in CRC development. ${ }^{52-54}$ Existing data suggest that their modulation can result in anticancer effects..$^{55}$ Thus, they may be more important as a therapeutic rather than diagnostic strategy.

\section{Endoscopy-Based Biomarkers}

Although stool- or blood-based methods remain most attractive, substantial efforts continue to be devoted to endoscopy-based screening and/or risk stratification methods. The endoscopic identification of raised lesions in the colorectum is typically straightforward. However, white light-driven detection of flat polyps or lesions can be more difficult, especially in a field of chronically inflamed intestinal mucosa (eg, ulcerative colitis). Topical chromoendoscopy, in which color dyes are applied directly to highlight mucosal variation, or virtual chromoendoscopy, in which light processing software built into high-definition endoscopes accomplishes a similar task, are well described. Standard chromoendoscopy was recently supported as the approach of choice in screening patients with ulcerative colitis. ${ }^{56}$ However, the feasibility of broad use has been questioned. Virtual chromoendoscopy implementation would be simpler; however, evidence to date is mixed about how much this technique augments standard highdefinition endoscopy to identify polyps. ${ }^{57,58}$

Novel endoscopic variations exploit other properties of light shined on intestinal mucosa. For example, light of certain wavelengths, in addition to being absorbed or reflected, can produce either autofluorescence $^{59}$ or stimulate a fluorescent response from exogenously introduced fluorophores. ${ }^{60}$ Variation in fluorescence patterns between normal and abnormal intestinal mucosa, including neoplastic change, are well described. These types of molecular imaging represent areas of intense research, but to date have not yielded clinically important advantages. Finally, some groups have attempted to exploit the possibility of field carcinogenesis, the concept that endogenous and exogenous factors conspire to create an environment permissive to widespread mutation (here, the entire colorectal mucosa). Techniques to evaluate mucosa for field defect could serve to stratify cancer risk across the entire bowel, not just the segment where sampling was performed. Early efforts with low-coherence enhanced backscattering spectroscopy have provided hopeful results that office-based interrogation of the rectal mucosa can predict the presence of neoplasia throughout the colorectum. ${ }^{61}$

\section{Conclusions}

This review surveys a wide range of CRC biomarkers in various stages of development. A few biomarker tests have reached the commercial stage. Identification of suitable risk stratification biomarkers could potentially increase the rate of CRC screening. If their predictive values are deemed adequate in specific clinical settings, they may offer attractive alternatives to colonoscopy-predominant screening strategies.

\section{References}

1. U.S. Cancer Statistics Working Group. United States cancer statistics: 1999-2012 incidence and mortality web-based report. Atlanta, GA: US Department of Health and Human Services, National Cancer Institute, CDC; 2015. Available at: http://www.cdc.gov/uscs. Accessed June 27 2016.

2. Zauber AG, Lansdorp-Vogelaar I, Knudsen AB, et al. Evaluating test strategies for colorectal cancer screening: a decision analysis for the U.S. Preventive Services Task Force. Ann Intern Med 2008;149:659-669.

3. Howlader N, Noone AM, Krapcho M, et al, eds. SEER Cancer Statistics Review, 1975-2012, National Cancer Institute. Bethesda, M. Available at: http://seer.cancer.gov/csr/1975_2012. Accessed June 27, 2016.

4. Centers for Disease Control and Prevention. Vital signs: colorectal cancer screening test use-United States, 2012. MMWR Morb Mortal Wkly Rep 2013;62:881-888

5. Imperiale TF, Monahan PO, Stump TE, et al. Derivation and validation of a scoring system to stratify risk for advanced colorectal neoplasia in asymptomatic adults: a cross-sectional study. Ann Intern Med 2015;163:339-346.

6. Zhu MM, Xu XT, Nie F, et al. Comparison of immunochemical and guaiac based fecal occult blood test in screening and surveillance for advanced colorectal neoplasms: a meta-analysis. J Dig Dis 2010;11:148-160.

7. Robinson MH, Pye G, Thomas WM, et al. Haemoccult screening for colorectal cancer: the effect of dietary restriction on compliance. Eur J Surg Oncol 1994;20:545-548.

8. Lee JK, Liles EG, Bent S, et al. Accuracy of fecal immunochemical tests for colorectal cancer: systematic review and meta-analysis. Ann Intern Med 2014;160:171.

9. Morikawa T, Kato J, Yamaji Y, et al. A comparison of the immunochemical fecal occult blood test and total colonoscopy in the asymptomatic population. Gastroenterology 2005;129:422-428.

10. Guittet L, Bouvier V, Mariotte N, et al. Comparison of a guaiac and an immunochemical faecal occult blood test for the detection of colonic lesions according to lesion type and location. Br J Cancer 2009;100:1230 1235.

11. Xu Y, Levy BT, Daly JM, et al. Comparison of patient preferences for fecal immunochemical test or colonoscopy using the analytic hierarchy process. BMC Health Serv Res 2015;15:175-183. 
12. Quintero E, Castells A, Bujanda L, et al. Colonoscopy versus fecal immunochemical testing in colorectal-cancer screening. N Engl J Med 2012;366:697-706.

13. Inadomi JM, Vijan $\mathrm{S}$, Janz $\mathrm{NK}$, et al. Adherence to colorectal cancer screening: a randomized clinical trial of competing strategies. Arch Intern Med 2012;172:575-582.

14. Ahlquist DA, Harrington JJ, Burgart LJ, Roche PC. Morphometric analysis of the "mucocellular layer" overlying colorectal cancer and normal mucosa: relevance to exfoliation and stool screening. Human Pathol 2000;31:5157.

15. Pritchard CC, Grady WM. Colorectal cancer molecular biology moves into clinical practice. Gut 2011;60:116-129.

16. Vogelstein B, Fearon ER, Hamilton SR, et al. Genetic alterations during colorectal-tumor development. N Engl J Med 1988;319:525-532.

17. Smith G, Carey FA, Beattie J, et al. Mutations in APC, Kirsten-ras, and p53-alternative genetic pathways to colorectal cancer. Proc Natl Acad Sci USA 2002;99:9433-9438.

18. Perucho M. Microsatellite instability: the mutator that mutates the other mutator. Nat Med 1996;2:630-631

19. Goldstein NS. Serrated pathway and APC (conventional)-type colorecta polyps: molecular-morphologic correlations, genetic pathways, and implications for classification. Am J Clin Pathol 2006;125:146-153.

20. Sinicrope FA, Rego RL, Halling KC, et al. Prognostic impact of microsatellite instability and DNA ploidy in human colon carcinoma patients. Gastroenterology 2006;131:729-737.

21. Shen L, Toyota M, Kondo $\mathrm{Y}$, et al. Integrated genetic and epigenetic analysis identifies three different subclasses of colon cancer. Proc Nat Acad Sci USA 2007;104:18654-18659.

22. Lao W, Grady WM. Epigenetics and colorectal cancer. Nat Rev Gastroenterol Hepatol 2011;8:686-700

23. Worthley DL, Leggett BA. Colorectal cancer: molecular features and clinical opportunities. Clin Biochem Rev 2010;31:31-38.

24. Dickinson BT, Kisiel J, Ahlquist DA, Grady WM. Molecular markers for colorectal cancer screening. Gut 2015;64:1485-1494.

25. Luo Y, Yu M, Grady WM. Field cancerization in the colon: a role for aberrant DNA methylation? Gastroenterol Rep (Oxf) 2014;2:16-20.

26. Xue M, Lai SC, Xu ZP, Want LJ. Noninvasive DNA methylation biomarkers in colorectal cancer: a systematic review. J Digest Diseases 2015;16:699-712.

27. Ned RM, Melillo S, Marrone M. Fecal DNA testing for colorectal cancer screening: the ColoSure test. PLoS Curr 2011;3:RRN1220.

28. Imperiale TF, Ransohoff DF, Itzkowitz SH, et al. Fecal DNA versus occult blood for colorectal cancer screening in an average-risk population. N Engl J Med 2004;351:2704-2714

29. Ahlquist DA, Zou H, Domanico M, et al. Next-generation stool DNA test accurately detects colorectal cancer and large adenomas. Gastroenterology 2012;142:248-256

30. Lidgard GP, Domanico MJ, Bruinsma JJ, et al. Clinical performance of an automated stool DNA assay for detection of colorectal neoplasia. Clin Gastroenterol Hepatol 2013;11:1313-1318.

31. Imperiale TF, Ransohoff DF, Itzkowitz $\mathrm{SH}$, et al. Multitarget stool DNA testing for colorectal-cancer screening. N Engl J Med 2014;370:1287-1297.

32. Hoff G, Grotmol T, Thiis-Evensen E, et al. Testing for faecal calprotectin (PhiCal) in the Norwegian Colorectal Cancer Prevention trial on flexible sigmoidoscopy screening: comparison with an immunochemical test for occult blood (FlexSure OBT). Gut 2004;53:1329-1333.

33. Li R, Liu J, Xue H, Huang G. Diagnostic value of fecal tumor M2-pyruvate kinase for CRC screening: a systematic review and meta-analysis. Int J Cancer 2012;131:1837-1845.

34. Leen R, Seng-Lee C, Holleran G, et al. Comparison of faecal M2-PK and FIT in a population-based bowel cancer screening cohort. Eur J Gastroenterol Hepatol 2014;26:514-518.

35. Robertson DJ, Imperiale TF. Stool testing for colorectal cancer screening. Gastroenterology 2015;149:1286-1293.

36. Flemer B, Lynch DB, Brown JM, et al. Tumour-associated and non-tumourassociated microbiota in colorectal cancer. Gut 2016;0:1-11.

37. Dulal S, Keku TO. Gut microbiome and colorectal adenomas. Cancer J 2014;20:225-231

38. Yu J, Feng Q, Wong SH, et al. Metagenomic analysis of faecal microbiome as a tool towards targeted non-invasive biomarkers for colorectal cancer [published online ahead of print September 25, 2015]. Gut, doi: 10.1136/ gutjnl-2015-309800.
39. Zeller G, Tap J, Voigt AY, et al. Potential of fecal microbiota for early-stage detection of colorectal cancer. Mol Syst Biol 2014;10:766.

40. Zackular JP, Baxter NT, Iverson KD, et al. The gut microbiome modulates colon tumorigenesis. MBio 2013;4:e00692-13.

41. Payne SR. From discovery to the clinic: the novel DNA methylation biomarker mSEPT9 for the detection of colorectal cancer in blood. Epigenomics 2010;2:575-585.

42. FDA Executive Summary. Prepared for the March 26, 2014 meeting of the Molecular and Clinical Genetics Panel on Epi proColon. Available at: http://www.fda.gov/downloads/ AdvisoryCommittees/CommitteesMeetingMaterials/MedicalDevices/ MedicalDevicesAdvisoryCommittee/MolecularandClinicalGeneticsPanel/ UCM390221.pdf. Accessed June 27, 2016.

43. Pedersen SK, Baker RT, McEvoy A, et al. A two-gene blood test for methylated DNA sensitive for colorectal cancer. PLoS One 2015;10:e0125041.

44. Ganepola GA, Nizin J, Rutledge JR, Chang DH. Use of blood-based biomarkers for early diagnosis and surveillance of colorectal cancer. World J Gastrointest Oncol 2014;6: 83-97.

45. Tsouma A, Aggeli C, Lembessis P, et al. Multiplex RT-PCR-based detections of CEA, CK20 and EGFR in colorectal cancer patients. World J Gastroenterol 2010;16:5965-5974.

46. Marshall KW, Mohr S, Khettabi FE, et al. A blood-based biomarker panel for stratifying current risk for colorectal cancer. Int J Cancer 2010;126:1177-1186.

47. Cortez MA, Bueso-Ramos C, Ferdin J, et al. MicroRNAs in body fluidsthe mix of hormones and biomarkers. Nat Rev Clin Oncol 2011;8:467477.

48. Shen J, Stass SA, Jiang F. MicroRNAs as potential biomarkers in human solid tumors. Cancer Lett 2013;329:125-136.

49. Schetter AJ, Leung SY, Sohn JJ, et al. MicroRNA expression profiles associated with prognosis and therapeutic outcome in colon adenocarcinoma. JAMA 2008;299:425-436.

50. Wang $B$, Zhang $Q$. The expression and clinical significance of circulating microRNA-21 in serum of five solid tumors. J Cancer Res Clin Oncol 2012;138:1659-1666.

51. Shi $X$, Sun M, Liu H, et al. Long non-coding RNAs: A new frontier in the study of human diseases. Canc Lett 2013;339:159-166.

52. Ge Z, Chen Y, Liao X, et al. Overexpression of long noncoding RNA PCAT-1 is a novel biomarker of poor prognosis in patients with colorectal cancer. Med Oncol 2013;30:588.

53. Zhai $\mathrm{H}$, Fesler $\mathrm{A}$, Schee $\mathrm{K}$, et al. Clinical significance of long intergenic noncoding RNA-p21 in colorectal cancer. Clin Colorectal Cancer 2013;12:261-266.

54. Ling H, Spizzo R, Atlasi $Y$, et al. CCAT2, a novel noncoding RNA mapping to $8 \mathrm{q} 24$, underlies metastatic progression and chromosomal instability in colon cancer. Genome Res 2013;23:1446-1461.

55. Li CH, Chen Y. Targeting long non-coding RNAs in cancers: progress and prospects. Int J Biochem Cell Biol 2013;45:1895-1910.

56. Laine L, Kaltenbach T, Barkun A, et al. SCENIC international consensus statement on surveillance and management of dysplasia in inflammatory bowel disease. Gastroenterology 2015;148:639-651.e28.

57. Hoffman A, Sar F, Goetz M, et al. High definition colonoscopy combined with i-Scan is superior in the detection of colorectal neoplasias compared with standard video colonoscopy: a prospective randomized controlled trial. Endoscopy 2010;42:827-833.

58. Adler A, Pohl H, Papanikolaou IS, et al. A prospective randomised study on narrow-band imaging versus conventional colonoscopy for adenoma detection: does narrow-band imaging induce a learning effect? Gut 2008;57:59-64.

59. van den Broek FJ, Fockens P, Van Eeden S, et al. Clinical evaluation of endoscopic trimodal imaging for the detection and differentiation of colonic polyps. Clin Gastroenterol Hepatol 2009;7:288-295.

60. Park Y, Ryu YM, Jung Y, et al. Spraying quantum dot conjugates in the colon of live animals enabled rapid and multiplex cancer diagnosis using endoscopy. ACS Nano 2014;8:8896-8910.

61. Radosevich AJ, Mutyal NN, Eshein A, et al. Rectal optical markers for in vivo risk stratification of premalignant colorectal lesions. Clin Cancer Res 2015;21:4347-4355.

62. Faivre J, Dancourt V, Lejeune C, et al. Reduction in colorectal cancer mortality by fecal occult blood screening in a French controlled study. Gastroenterology 2004;126:1674-1680.

63. Shaukat A, Mongin SJ, Geisser MS, et al. Long-term mortality after screening for colorectal cancer. N Engl J Med 2013;369:1106-1114. 
64. Nishihara R, Wu K, Lochhead P, et al. Long-term colorectal-cancer incidence and mortality after lower endoscopy. N Engl J Med 2013;369:1095-1105.

65. Nguyen MT, Weinberg DS. The impact of colonoscopy on colorectal cancer incidence and mortality. In: Miskovitz P, ed. Colonoscopy. Rijeka, Croatia: INTECH; 2011:79-96. Available at: http://www.intechopen.com/ books/colonoscopy. Accessed June 27, 2016.
66. Johnson CD, Chen MH, Toledano AY, et al. Accuracy of CT colonography for detection of large adenomas and cancers. N Engl J Med 2008;359:12071217.

67. Pickhardt PJ, Choi JR, Hwang I, et al. Computed tomographic virtual colonoscopy to screen for colorectal neoplasia in asymptomatic adults. N Engl J Med 2003;349:2191-2200.

\section{Instructions for Completion}

To participate in this journal CE activity: 1) review the learning objectives and author disclosures; 2 ) study the education content; 3 ) take the posttest with a $66 \%$ minimum passing score and complete the evaluation at http://education.nccn.org/ node/79280; and 4) view/print certificate. After reading the article, you should be able to answer the following multiple- choice questions. Credit cannot be obtained for tests completed on paper. You must be a registered user on NCCN.org. If you are not registered on NCCN.org, click on "New Member? Sign up here" link on the left hand side of the Web site to register. Only one answer is correct for each question. Once you successfully answer all posttest questions you will be able to view and/or print your certificate. Software requirements: Internet

\section{Posttest Questions}

1. CRC detected through screening has approximately a 5 -year survival rate.
a. $65 \%$
b. $80 \%$
c. $90 \%$

2. Stool DNA methylation biomarkers have all of the following advances over similar blood biomarkers except:

a. Aberrant DNA methylation in the blood may signal the presence of different cancers at the same time

b. Early CRC or precursor lesion may not yet release markers into the bloodstream

c. mFBN1 and VIM in feces can be identified in early-stage CRC d. Numerous hypermethylated genes have been detected in blood samples more readily than in human stool

3. Next-generation sequencing:

a. Enables researchers to perform direct RNA-seq to sequence the whole transcriptome

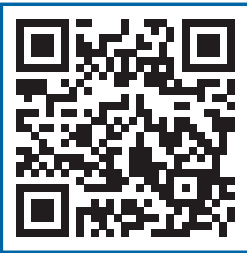

b. Is less costly than mRNA expression microarray profiles and validation using RT-qPCR

c. Is convenient (eg, faster than RT-qPCR)

d. Both $a$ and $b$

e. All of the above 